Document downloaded from:

http://hdl.handle.net/10251/99734

This paper must be cited as:

Lofland, S.; García Miquel, ẢH.; Vázquez, M.; Bhagat, S. (2002). Microwave magnetoabsorption in glass-coated amorphous microwires with radii close to skin depth. Journal of Applied Physics. 92(4):2058-2063. doi:10.1063/1.1494847

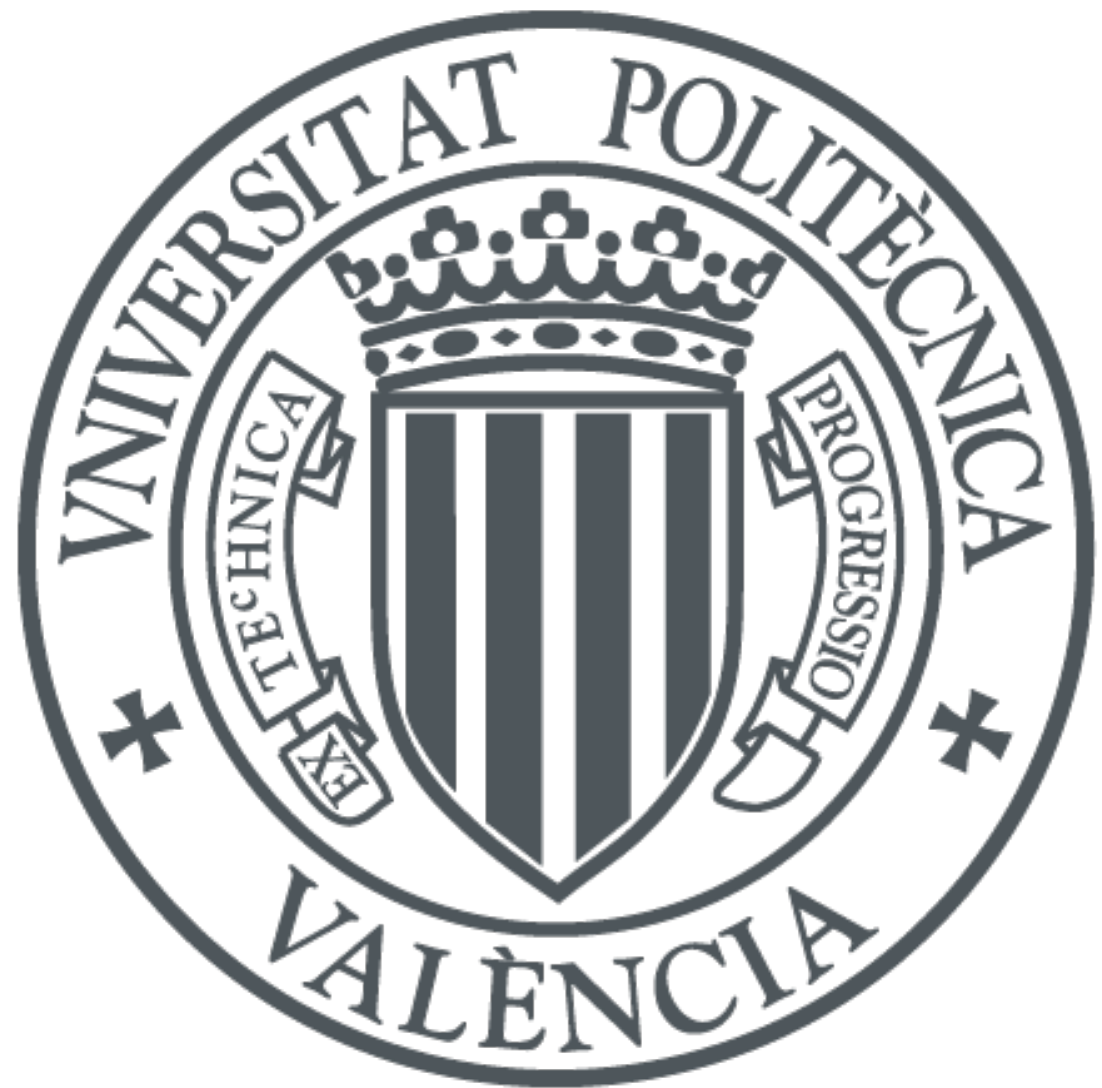

The final publication is available at https://doi.org/10.1063/1.1494847

Copyright American Institute of Physics

Additional Information 


\title{
Microwave magnetoabsorption in glass-coated amorphous microwires with radii close to skin depth
}

\author{
S. E. Loflan a) \\ Department of Physics, Ferromagnetic Resonance Group, University of Maryland, College Park, \\ Maryland 20742-4111 and Department of Chemistry and Physics, Rowan University, New Jersey \\ 08028-1701 \\ H. Garcia-Miquel \\ Department of Physics, Ferromagnetic Resonance Group, University of Maryland, College Park, \\ Maryland 20742-4111 and Departamento de Ingenieria Electroniea, Universidad Politecnica de Valencia, \\ Camino de Vera sn, 46021 Valencia, Spain
}

M. Vazquez

Instituto de Ciencia de Materiales, CSIC, 28049 Cantoblanco, Madrid, Spain

S. M. Bhagat

Department of Physics, Ferromagnetic Resonance Group, University of Maryland, College Park, Maryland 20742-4111

()

We present the frequency dependence of microwave magnetoabsorption in glass-coated amorphous microwires of $\left(\mathrm{Co}_{100-x} \mathrm{Fe}_{x}\right)_{72.5} \mathrm{Si}_{12.5} \mathrm{~B}_{15}$. The data were taken at room temperature in the frequency range of $1-60 \mathrm{GHz}$ for field up to $15 \mathrm{kOe}$ by either a cavity perturbation technique or a coaxial transmission line. The resulting spectra strongly depend upon the local microwave magnetic and electric fields We have found that we can simulate the spectra using an analytic solution to the problem of electromagnetic scattering from a cylinder. We demonstrate that these unusual spectra can be interpreted in terms of ferromagnetic resonance and antiferromagnetic resonance. However, because the electromagnetic skin depth is comparable to the radius, the resonance and antiresonance field do not follow the conventional equations.

[DOI: $10.1063 / 1.1494847$ ]

\section{INTRODUCTION}

During the past few years, there has been increasing interest in amorphous magnetic microwires as they exhibit giant magnetoimpedance ${ }^{1,2}$ at low field and can also display unusual domain structures ${ }^{3}$ because of the large strains frozen in during the manufacturing process. Since some of these properties make them useful for microwave devices, a careful study is essential to establish appropriate design criteria. When they are studied by techniques such as ac susceptibility and dc magnetization, obtaining accurate values for magnetic properties such as the magnetization becomes challenging since the sample dimensions need to be known rather precisely. Ferromagnetic resonance (FMR) measurements, on the other hand, require knowledge of only the sample shape to determine the magnetic parameters. In addition, they will serve to determine the sources of spin relaxation as well as reveal magnetic inhomogeneities, if any.

Previous FMR studies of microwires ${ }^{4-6}$ have not always been without controversy regarding the interpretation of the spectra. In order to help settle some of these issues, we undertook a systemic experimental and theoretical investigation of magnetoabsorption in microwires covering a wide range of frequencies and samples having a broad span of magne-

\footnotetext{
a) Author to whom correspondence should be addressed; electronic mail: lofland@rowan.ed
}

tostriction constants. As we shall see, the observed spectra are far from being conventional. The interpretation requires careful analysis of the interaction between the sample and the electromagnetic fields In some sense, the situation is rather similar to that found in early experiments on ferrite spheres. ${ }^{7}$ In both cases the crucial point is that the sample size is comparable to the electromagnetic skin depth.

\section{METHODS}

Glass-clad microwires of $\left(\mathrm{Co}_{100-x} \mathrm{Fe}_{x}\right)_{0.725} \mathrm{Si}_{12,5} \mathrm{~B}_{15}$ with $0<x<100$ were prepared by the Taylor-Ulitovsky method. ${ }^{8}$ The series consisted of 15 samples with $x$ $=\{0,2,4,6,8,10,20,30,40,50,60,70,80,90,100\}$. Near $x=0$, the Fe content was increased in small steps in order to study the effect of the transition from negative to positive magnetostriction at about $6 \% \mathrm{Fe} .{ }^{9}$ In what follows, the samples are designated $\mathrm{M} x$, where $x$ represents the percentage of Fe. The nominal radius of the studied microwires is $1.5-3 \mu \mathrm{m}$. However, the method of manufacture does not allow one to control the radius precisely. Thus, there will be significan variation in radius along the length of the wire. As we shall see, this interferes with a precise determination of the materials properties.

Microwave studies were done by two methods. The firs was a cavity-perturbation technique done at frequencies $f$ $=9.7,26.7,32.7$, and $56 \mathrm{GHz}$ with conventional homodyne 
detection. A single wire was located in the cavity such that the rf electric fiel $e_{\mathrm{rf}}$ was nearly zero. This is very important. The dc fiel $H(0<H<16 \mathrm{kOe})$ was along the axis of the wire while the rf magnetic fiel $h_{\mathrm{rf}}$ was perpendicular to it. Since many of the spectra contained fairly broad features, fiel modulation was not employed.

The second used a terminated coaxial transmission line for $1 \mathrm{GHz}<f<8 \mathrm{GHz}$ which was discussed in detail in Ref. 10. To summarize, we used a modifie transmission line wherein the dielectric was replaced by several microwires aligned axially rather than the single wire used in the cavity perturbation method, and $H$ was applied along the "sample." In this situation, both the high frequency field $e_{\mathrm{rf}}$ and $h_{\mathrm{rf}}$ are nonzero at the sample. The transmission line was terminated with a short so that the reflectio parameter provided a determination of the magnetoabsorption as the frequency was swept, holding $H$ constant, in contrast to the cavity perturbation technique where $H$ was swept.

\section{RESULTS}

By and large, wires for all $x$ values exhibit the same spectral features. Two typical sets of spectra $(x=10,80)$ taken from the cavity method are shown in Figs. 1(a) and 1 (b), respectively. It is notable that at $9.7 \mathrm{GHz}$ one observes only a broad (several hundred Oe wide) dip in the absorption. That is, a signal reminiscent of the ferromagnetic antiresonance (FMAR) ${ }^{11}$ is the only observable feature. At the higher frequencies, one can identify an absorption peak. However, it is severely distorted in that the signal drops sharply just above the peak and exhibits a weak minimum prior to becoming fiel independent at high fields In addition, one should note that: (a) the observed spectral shapes are rather sensitive to the location of the conducting sample in the cavity. One has to ensure that the loss due to $e_{\mathrm{rf}}$ does not become dominant (this is particularly important for $f$ $\leqslant 10 \mathrm{GHz}$ ); (b) many wires exhibit more than one poorly resolved line; (c) for given $x$, the spectrum is not exactly the same for every piece of the wire, reflectin the possible inhomogeneities consequent on the manufacturing process; and (d) the wire radius (an important parameter, see Fig. 4) tends to vary somewhat.

\section{DISCUSSION}

It is apparent from Fig. 1 that FMR is not only unobservable at $f<10 \mathrm{GHz}$ but also when it does appear at higher $f$ it is rather distorted. Therefore, it is very surprising that the characteristic field $H_{1}$ and $H_{2}$ follow what appear to be FMR conventional equations

$$
\left(\frac{\omega}{\gamma}\right)^{2}=\left(H_{1}+H_{\mathrm{an}}\right)\left(H_{1}+H_{\mathrm{an}}+4 \pi M_{\mathrm{eff}}\right)
$$

for the fiel in the plane of a disk

$$
\frac{\omega}{\gamma}=\left(H_{2}+H_{\mathrm{an}}+2 \pi M_{\mathrm{eff}}\right)
$$

for a cylinder with the fiel parallel to the axis. The effective parameter values for various samples are listed in Table I. Here, the symbols have their usual meanings; $\omega$ is the angu-
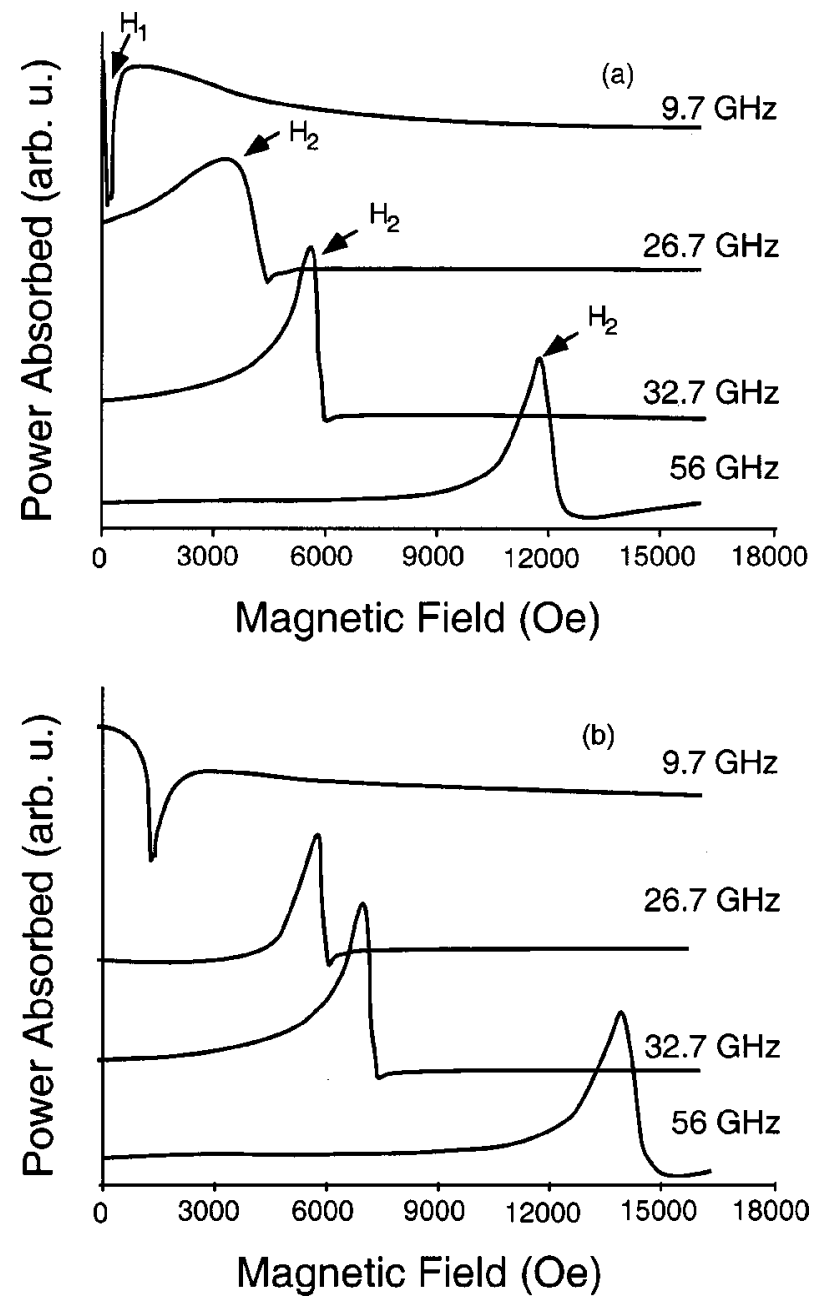

FIG. 1. Spectra for: (a) $x=80$ and (b) $x=10$ at various frequencies taken by the cavity perturbation methods. At $9.7 \mathrm{GHz}$, there is only a dip while at higher frequencies there are both a maximum and a minimum.

lar frequency, $\gamma=g \mu_{B} \hbar$ the gyromagnetic ratio, $M_{\text {eff }}$ the effective saturation magnetization, and $H_{\text {an }}$ the anisotropy field $H_{\text {an }}$ is written with the implicit assumption that the symmetry axis is along the wire.

However, in light of the discussion below, and keeping in mind that the spectral features are rather wide, it is not too fruitful to compare these values to other determinations apart

TABLE I. Empirical parameters [Eqs. (1) and (2)].

\begin{tabular}{cccc}
\hline \hline$x$ & $g_{\text {eff }}$ & $\begin{array}{c}M_{\text {eff }} \\
\left(\mathrm{emu} / \mathrm{cm}^{3}\right)\end{array}$ & $\begin{array}{c}H_{\text {an }} \\
(\mathrm{Oe})\end{array}$ \\
\hline 0 & 2.23 & 440 & 0 \\
10 & 2.26 & 480 & 110 \\
20 & 2.36 & 450 & 250 \\
30 & 2.27 & 620 & 300 \\
40 & 2.27 & 580 & 200 \\
50 & 2.20 & 850 & 370 \\
60 & 2.20 & 770 & 340 \\
70 & 2.18 & 820 & 340 \\
80 & 2.27 & 800 & 320 \\
90 & 2.28 & 770 & 250 \\
100 & 2.30 & 690 & 260 \\
\hline \hline
\end{tabular}



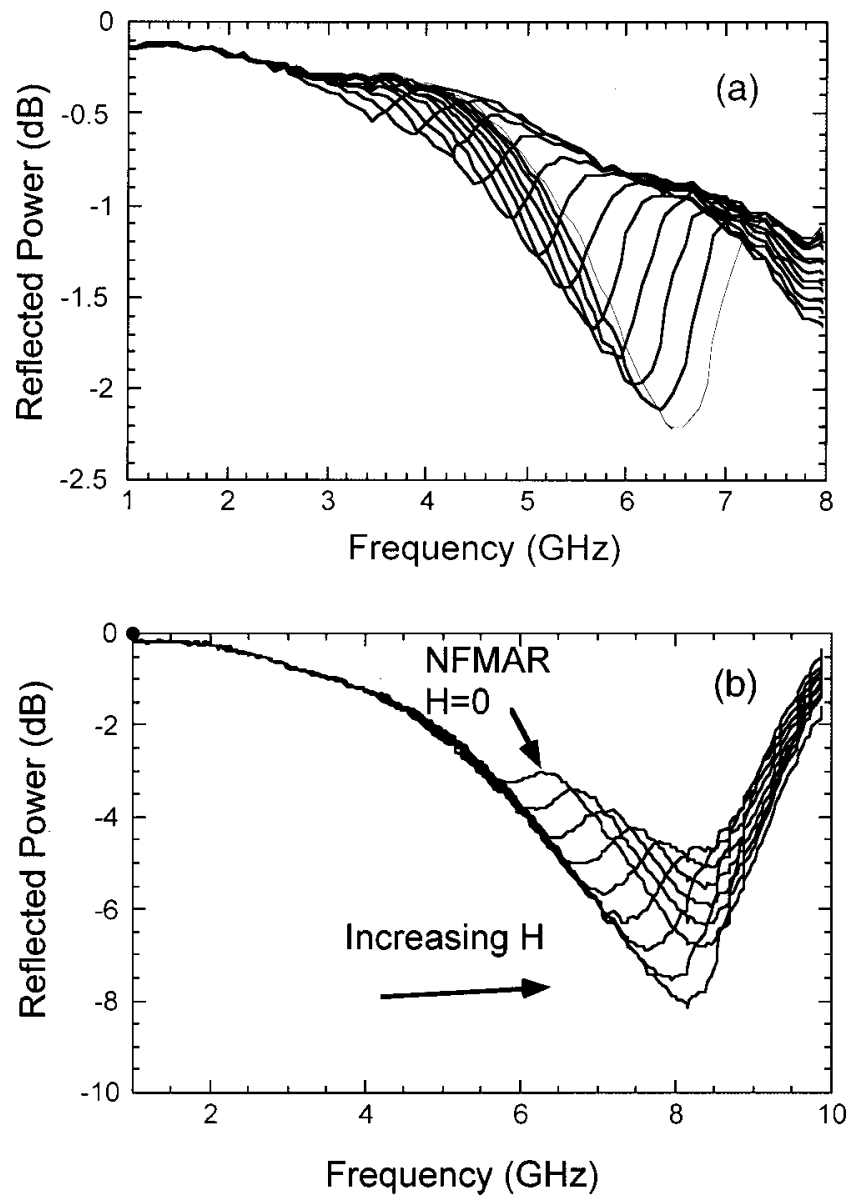

FIG. 2. Spectra for: (a) $x=0$ and (b) $x=60$ taken as a function of frequency at constant fiel by the coaxial line technique. In all cases, one observes a peak. Note for (b), there is even a peak at $H=0$.

from remarking that the $M_{\text {eff }}$ values are not too different from the dc magnetization data except at the highest $x$ and the $H_{\text {an }}$ values can be reasonably ascribed to magnetoelastic effects with credible values for the frozen-in stress ${ }^{12}$ and the magnetostriction constants. ${ }^{9}$

In a recent article, ${ }^{6}$ Eq. (1) was used to interpret what were claimed to be FMR spectra in the present samples at $f \leqslant 8 \mathrm{GHz}$ (Fig. 2). Although the data appeared to yield reasonable values for $H_{\text {an }}$ and $M$, the analysis is not justifie since the electromagnetic skin depth $\delta=\delta_{0} / \sqrt{\mu}\left[\delta_{0}\right.$ $=c / \sqrt{2 \pi \omega \sigma} \approx(16 \mu \mathrm{m}) / \sqrt{f / \mathrm{GHz}}]$, where $c$ is the speed of light and $\sigma$ the conductivity $\left(\sigma^{-1} \sim 100 \mu \Omega \mathrm{cm}\right.$ ) is always much larger than the wire radius $a$. Indeed, as noted above, the low-frequency feature should be interpreted as a transparency or FMAR (Fig. 1). Further details of the low- $f$ data are discussed later. The question is, why is Eq. (1) appropriate?

To be precise the main point is that when $a \sim \delta_{0}$, the dynamic variables $\mathbf{m}$ and $\mathbf{h}_{\mathrm{rf}}$ are not uniform inside the sample and the use of dynamic demagnetization factors is inappropriate. Rather, one must use the generalized permeability tensor $\vec{\mu}$ and explicitly invoke the boundary conditions on both the dynamic magnetic induction $\mathbf{b}_{\mathrm{rf}}$ and magnetic fiel $\mathbf{h}_{\mathrm{rf}}$ at the sample surface so as to take account of the variation of the dynamic field inside the cylinder. Since we are considering the case where the dc fiel is applied along the cylinder $(z)$ axis, the cylindrical symmetry of $\overleftrightarrow{\mu}$

$$
\overleftrightarrow{\mu}=\left[\begin{array}{ccc}
\mu_{1} & -i \mu^{\prime} & 0 \\
i \mu^{\prime} & \mu_{1} & 0 \\
0 & 0 & \mu_{z}
\end{array}\right]
$$

allows for an analytic solution to the problem of a plane wave incident on a cylinder, the general form firs given by Sammadar. ${ }^{13}$ The plane-wave approximation seems reasonable for cavity perturbation results as we used conventional microwave spectrometers, with $\mathrm{TE}_{10 n}$ mode rectangular cavities with the wire in a location where the rf electric fiel is very nearly zero.

We assume that, as a firs approximation, we may ignore the damping and use the Polder permeability ${ }^{14}$

$$
\vec{\mu}=\left[\begin{array}{ccc}
\frac{\omega^{2}-\gamma^{2} H(H+4 \pi M)}{\omega^{2}-\gamma^{2} H^{2}} & \frac{-4 \pi i M \omega \gamma}{\omega^{2}-\gamma^{2} H^{2}} & 0 \\
\frac{4 \pi i M \omega \gamma}{\omega^{2}-\gamma^{2} H^{2}} & \frac{\omega^{2}-\gamma^{2} H(H+4 \pi M)}{\omega^{2}-\gamma^{2} H^{2}} & 0 \\
0 & 0 & 1
\end{array}\right] .
$$

We take the permittivity $\varepsilon$ to be isotropic with $\varepsilon$ $=4 \pi i \sigma / \omega$. The case considered here is one of normal incidence with the incident $\mathbf{h}_{\mathrm{rf}}$ being perpendicular to the cylinder axis. Following Ref. 13, the scattered moments $A_{n}$ are written as

$$
\begin{aligned}
A_{n}^{s}= & i^{n} \frac{J_{n}(k a)}{H_{n}^{(2)}(k a)} \\
& \times\left[\frac{\frac{n}{k_{1} a} \frac{k}{k_{1}} \frac{\mu^{\prime}}{\mu_{1}}+\frac{k}{k_{1}} \frac{J_{n}^{\prime}\left(k_{1} a\right)}{J_{n}\left(k_{1} a\right)}-\frac{J_{n}^{\prime}(k a)}{J_{n}(k a)}}{\mu_{1} a} \frac{k}{k_{1}} \frac{J_{n}^{\prime}\left(k_{1} a\right)}{k_{1}}-\frac{H_{n}^{\prime(2)}(k a)}{J_{n}\left(k_{1} a\right)}\right.
\end{aligned}
$$

with

$$
k_{1}=\frac{\varepsilon}{\mu_{1}} k \sqrt{\mu_{1}^{2}-\mu^{\prime 2}},
$$

where $J_{n}(x)$ is the Bessel function of order $n, H_{n}^{(2)}(x)$ the Hankel function of the second kind of order $n$ with primes indicating the derivative of the function with respect to its argument, and $k=\omega / c$ is the freespace wave number. Since the sample radii are much smaller than the freespace wavelength $2 \pi / k$, the relevant quantity is the dipole moment per unit length with the microwave absorption being proportional to its imaginary part. Thus, we need only to sum the $n=1$ and -1 terms of the scattered moments. That is, the power absorption $P$ is given by

$$
P \propto \operatorname{Im}\left(A_{s}^{-1}+A_{s}^{1}\right) .
$$

We have calculated the spectra using $\delta_{0}$ $=(16 \mu \mathrm{m}) / \sqrt{f / \mathrm{GHz}}, \quad M=1000 \mathrm{emu} / \mathrm{cm}^{3}, \quad g=2.2$, and $a$ $=2.5 \mu \mathrm{m}$, appropriate to $x=80$. Note that qualitatively, the computed spectra of Fig. 3 are in excellent agreement with the experimental results of Fig. 1(a). 


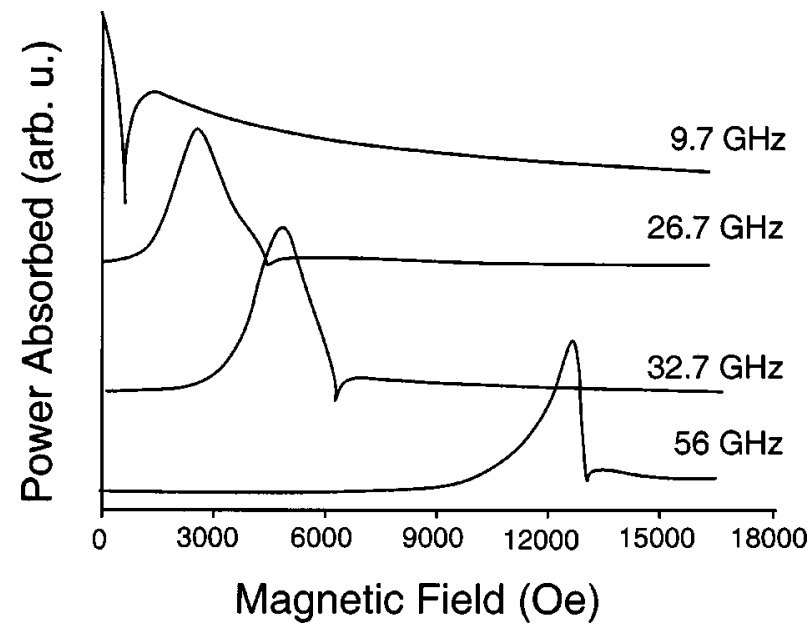

FIG. 3. Calculated spectra for $x=80$, using $M=1000 \mathrm{emu} / \mathrm{cm}^{3}, g=2.2$, and $a=2.5 \mu \mathrm{m}$. There are qualitative similarities between the simulations and the spectra in Fig. 1.

At low frequencies, there is only a FMAR. This occurs at the dc fiel where the diagonal term in the permeability is zero, namely $\left[(\omega / \gamma)^{2}=H(H+4 \pi M)\right]$, which is identical to Eq. (1). Note that $b_{\text {rf }}$ vanishes at this value of $H$. Hence, the induced electric fiel and concomitantly $P$ vanish. For very low frequencies or for very thin wires, where $\delta$ is much larger than $a$, the transparency will be unobservable since the eddy current losses are small in any case. For $\omega>2 \pi \gamma M$, in addition to the FMAR, FMR should appear at

$$
\frac{\omega}{\gamma} \approx H+2 \pi M \text {. }
$$

The approximate sign reflect the effect of the sample size, which is discussed next.

In Fig. 4, we have calculated the spectra for $26.7 \mathrm{GHz}$ using the aforementioned values for $g$ and $M$ with $a$ varying from 1 to $3.5 \mu \mathrm{m}$. There is a significan change in the spectrum with $a$ mainly because the resonance fiel varies with $a$.

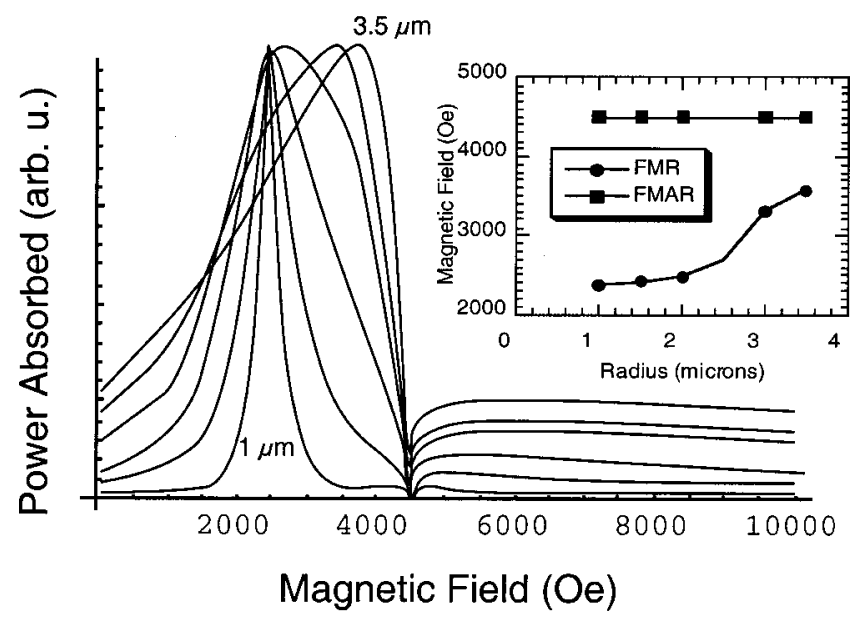

FIG. 4. Calculated spectra, using $M=1000 \mathrm{emu} / \mathrm{cm}^{3}, g=2.2$, and $26.7 \mathrm{GHz}$ for various values of $a$ with $\delta_{0} \sim 3 \mu \mathrm{m}$. The spectra are rather sensitive to the radius for $a \sim \delta$. The inset shows the calculated dependence of the field for $\operatorname{FMAR}\left(H_{1}\right)$ and $\operatorname{FMR}\left(H_{2}\right)$ on radius.

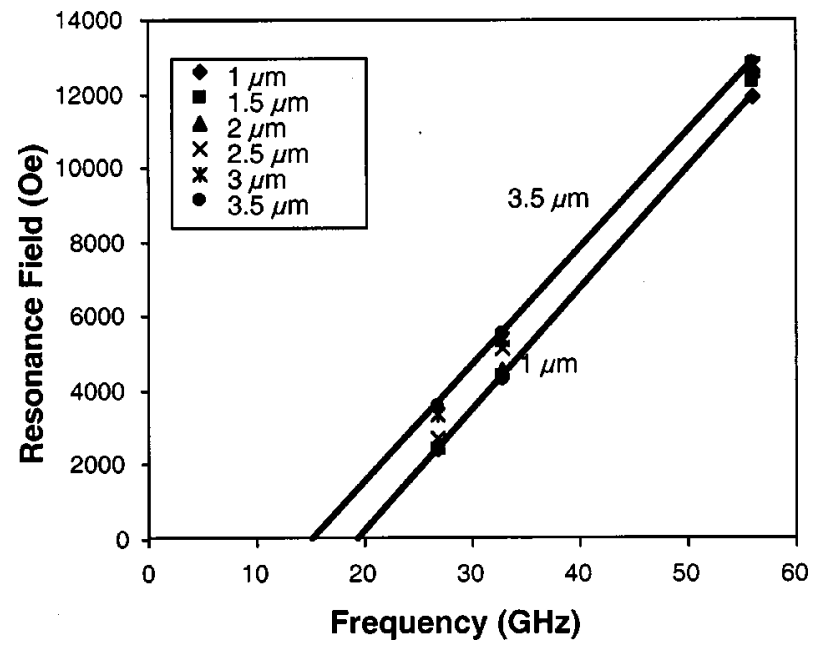

FIG. 5. Frequency dependence of the resonance field using $M$ $=1000 \mathrm{emu} / \mathrm{cm}^{3}, g=2.2$, and $26.7 \mathrm{GHz}$ for various values of $a$. If one assumes Eq. (4) is the proper interpretation of the data, the resulting values for $g$ and $M$ vary significantl .

This is due to the fact that as $a$ becomes comparable to $\delta_{0}$, the resonance fiel must eventually change to that expected of a parallel plate. Consequently the FMR lines are distorted. The shift from the value given by Eq. (8) is quite sizable, even for $a=1 \mu \mathrm{m}$. Note, however, that the FMAR does not move (Fig. 4, inset).

In Fig. 5, we plot the calculated resonance field as a function of frequency for $a$ varying from 1 to $3.5 \mu \mathrm{m}$. If one were to assume that Eq. (8) is appropriate to interpret the FMR, the resulting slopes and intercepts would vary considerably, and at best one would obtain effective $g$ values lying between 2.1 and 2.3 and effective $M$ values ranging from 760 to $1080 \mathrm{emu} / \mathrm{cm}^{3}$ (cf. Table I). Obviously, without a precise knowledge of $a$ and $\sigma$, any determination of the magnetic parameters will be far from precise.

To repeat, while the simulated curves qualitatively reproduce the features in the measured spectra, it is not fruitful to attempt a quantitative match to fi the material parameters. At this stage, further caveats must be mentioned. In addition to concerns about sample homogeneity, as well as precise knowledge of sample size and resistivity, there are other issues. For example, as shown by Jackson and Barmatz, ${ }^{15}$ the field in a rectangular cavity can be represented as the superposition of six plane waves. We have used the solution of a single plane wave scattering off a cylinder as an approximation to the cavity perturbation problem. This seems to be reasonable for the present situation. Since we have assumed that $\mathbf{h}_{\mathrm{rf}}$ is normal to the cylinder axis and because we have a conducting cylinder, $\mathbf{e}_{\mathrm{rf}}$ must be near zero to satisfy the boundary conditions. This is close to the situation under which the measurements were made. However, in general, one notes that slight misalignment of the sample within the cavity may cause $\mathbf{e}_{\mathrm{rf}}$ to be large at the sample surface, a situation which cannot be realized by considering a plane wave scattering off a conducting surface.

While the fiel values of the features within the spectra are likely to be unaffected, their shapes are rather sensitive to the relative values of $\mathbf{h}_{\mathrm{rf}}$ and $\mathbf{e}_{\mathrm{rf}}$ A single microwire was 


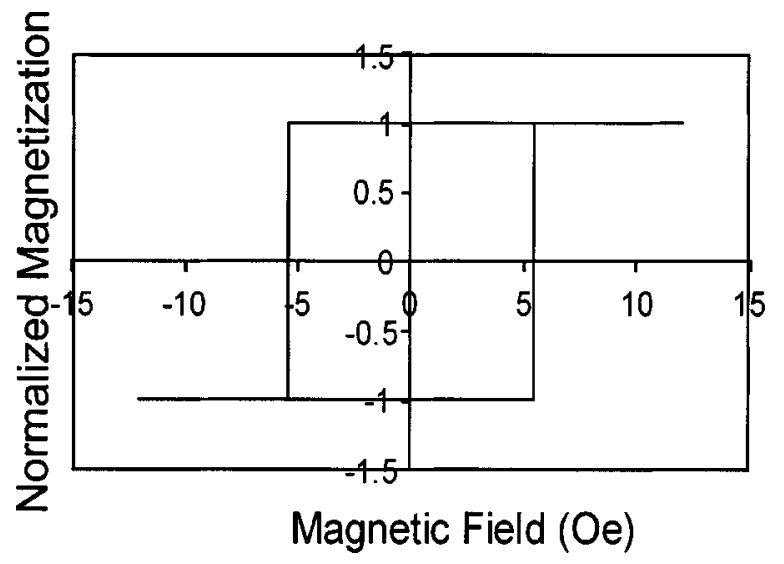

FIG. 6. Field dependence of the magnetization of $x=60$. The sample is bistable at $H=0$.

studied by the cavity perturbation technique, using a rectangular cavity at $9.5 \mathrm{GHz}$ and sweeping $H$. As discussed in earlier, if the sample was placed in such a way that $h_{\mathrm{rf}}$ was a maximum and $e_{\mathrm{rf}}$ nearly zero, one observed the expected dip in absorption due to FMAR. If, however, $e_{\mathrm{rf}}$ was nonzero, a peak in the absorption appeared at the same field In the absence of our latest findings it is understandable why in Ref. 6, some of us were led to claim that the peak in the absorption in Fig. 2 be ascribed to FMR. As noted above, the observed spectra are extremely sensitive to the placement of the sample.

The results of the cavity perturbation methods are critical in interpreting the data taken from the terminated coaxial line (Fig. 2). One complication is that the samples studied are not single wires and their placement is random so that variations in the local fiel are difficul to ascertain. Nevertheless, it is believed that to a firs approximation the essential physics can be accessed with the analysis that we have discussed. One notes that under appropriate conditions, it should be possible to observe a zero-fiel FMAR or natural (N) FMAR [Eq. (1)]. In light of the above discussion of sample placement, the peaks of Fig. 2 are now identifie as FMAR, and in Fig. 2(b) one also notes the NFMAR in $x$ $=60$ for $H=0$.

Figure 6 shows the hysteresis loop for $x=60$ and one notes the clear bistable behavior, with a very small coercive field The existence of a stable residual state accounts for the NFMAR in Fig. 2(b).

In Fig. 7 are collected together representative lowfrequency FMAR data for $x=0$ and 60. It is to be noted that $H \ll M$ for all the samples, thus Eq. (1) simplifie to

$$
\left(\frac{\omega}{\gamma}\right)^{2}=4 \pi M\left(H+H_{\mathrm{an}}\right)
$$

and provides an excellent description for all the observations. It is clear that because of the effects of sample size, there is considerable ambiguity regarding the values of the parameters $g$ and $M$ and concomitantly $H_{\text {an }}$ since we have no way of accessing the precise radius of every wire in the sample, which consists of many (10-20) microwires.

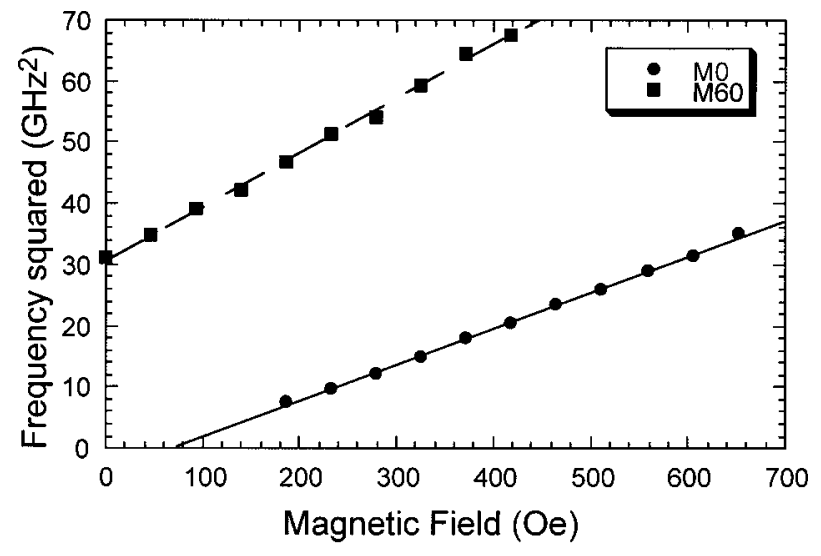

FIG. 7. Field dependence of the antiresonance frequency [Eq. (9)].

A rough idea of the $x$ dependence of $4 \pi M$ and $H_{\text {an }}$ can be obtained by picking a credible value for $g$, namely 2.2. If so, we get the results shown in Figs. 8(a) and 8(b). As expected, the "errors" are sizable, especially at large $x$. However, it is gratifying that $M$ increases roughly linearly with $x$ as also found by dc magnetization measurements ${ }^{9}$ and that values from the low-frequency (coaxial line, Fig. 8) and high-frequency (cavity perturbation, Table I) methods agree. As expected, $H_{\mathrm{an}} \sim 0$ for low $x$, where the magnetostriction constant $\lambda$ is rather small. For the most part, $H_{\mathrm{an}} \sim 3 \lambda \sigma / M$,
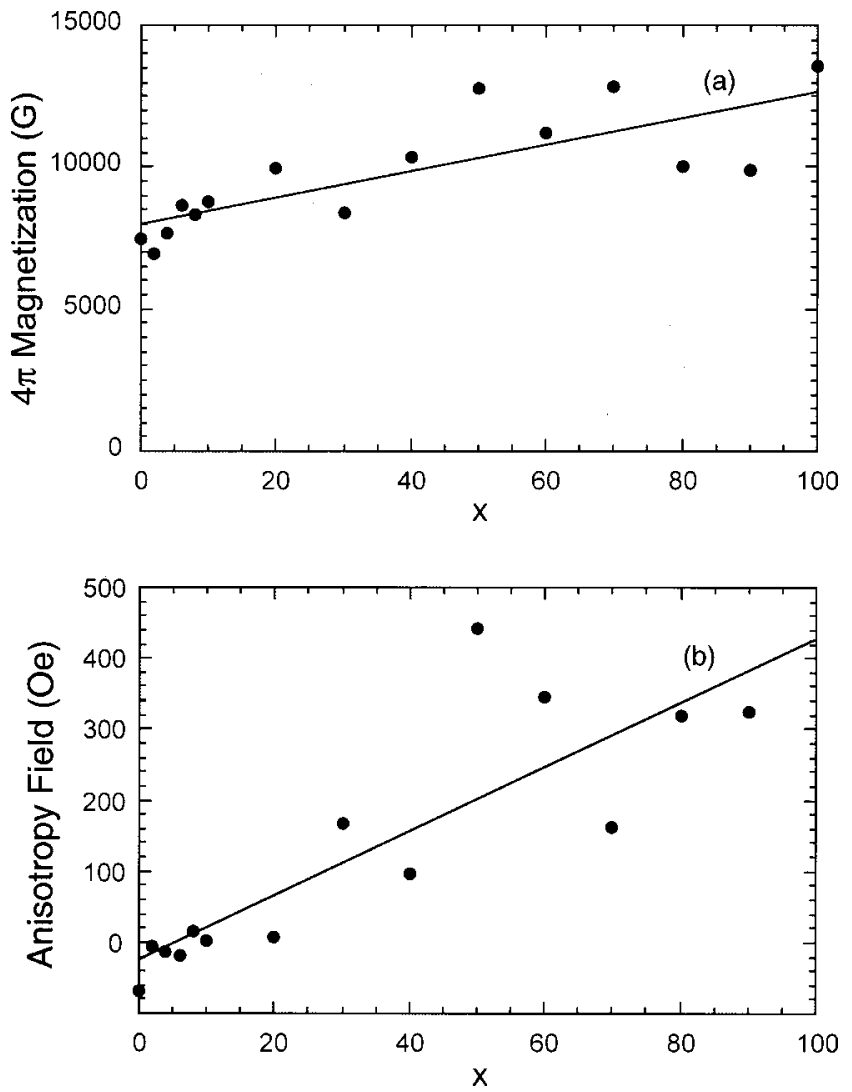

FIG. 8. Dependence of: (a) the magnetization and (b) anisotropy fiel on doping determined from the low-frequency data. These values are in accord with those taken at high frequency (Table I). 
where $\sigma$ is the stress, so it should be relatively insensitive to $x$ as $\lambda$ and $M$ both increase with $x$.

To conclude, a study of the magnetoabsorption of microwave radiation in micron-size amorphous microwires shows that if one wishes to use FMR to access the magnetic parameters precisely, measurements will have to be made at very high frequencies $(>150 \mathrm{GHz})$ in order to ensure that the electromagnetic skin depth is much smaller than the wire radius so that the conventional FMR equations become applicable. At lower frequencies, the dynamic demagnetizing factors become nonuniform and the resonance field become size dependent in a nontrivial manner.

\section{ACKNOWLEDGMENTS}

H.G.-M. wants to thank the "Ministerio de Educacion y Cultura" of Spain for a grant. S.E.L. acknowledges support by NSF MRSEC Grant No. DMR00-80008 and the New Jersey Commission on Higher Education.
${ }^{1}$ K. Mandal and S. K. Ghatak, Phys. Rev. B 47, 14233 (1993).

${ }^{2}$ F. L. A. Machado, B. Lopes da Silva, and E. Montarroyos, J. Appl. Phys. 73, 6387 (1993).

${ }^{3}$ O. Acher, P.-M. Jacquart, and C. Boscher, IEEE Trans. Magn. 30, 4542 (1994).

${ }^{4}$ A. Adenot et al., J. Appl. Phys. 87, 5965 (2000).

${ }^{5}$ M. R. Britel et al., Appl. Phys. Lett. 77, 2737 (2000).

${ }^{6}$ H. Garcia-Miquel, M. J. Esbri, J. M. Andres, J. M. Garcia, J. M. GarciaBeneytez, and M. Vazquez, IEEE Trans. Magn. 37, 561 (2001).

${ }^{7}$ W. A. Yager, J. K. Galt, and F. R. Merritt, Phys. Rev. 99, 1203 (1955).

${ }^{8}$ G. F. Taylor, Phys. Rev. 24, 655 (1924).

${ }^{9}$ M. Knobel, R. Sato Turtelli, and R. Grossinger, J. Magn. Magn. Mater. 116, 154 (1992).

${ }^{10}$ H. García-Miquel, J. M. García-Beneytez, and M. Vázquez, J. Magn. Magn. Mater. 231, 38 (2001).

${ }^{11}$ B. Heinrich and V. F. Mescheryakov, JETP Lett. 9, 378 (1969).

${ }^{12}$ J. Velazquez, M. Vazquez, and A. P. Zhukov, J. Mater. Res. 11, 2499 (1996).

${ }^{13}$ S. N. Samaddar, Appl. Sci. Res. 10, 385 (1963).

${ }^{14}$ D. Polder, Philos. Mag. 40, 99 (1949).

${ }^{15}$ H. W. Jackson and M. Barmatz, J. Appl. Phys. 70, 5193 (1991). 